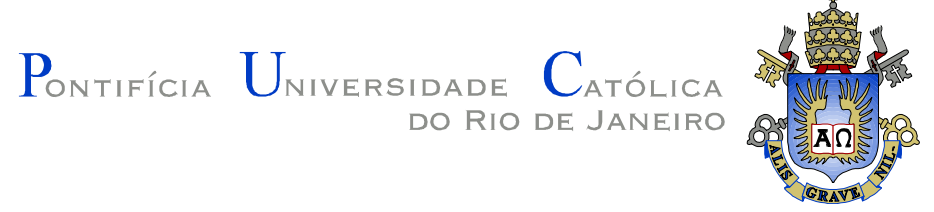

Luis Carlos Castillo Martínez

\title{
Otimização dos Circuitos de Refrigerante nos Trocadores de Calor de Sistemas de Refrigeração por Compressão de Vapor
}

Tese de Doutorado

Tese apresentada como requisito parcial para obtenção do título de Doutor pelo Programa de Pós-Graduação em Engenharia Mecânica da PUC-Rio.

Orientador: José Alberto dos Reis Parise Co-orientadores: Samuel Fortunato Yana Motta Elizabet del Carmen Vera Becerra 


\section{Otimização dos Circuitos de Refrigerante nos Trocadores de Calor de Sistemas de Refrigeração por Compressão de Vapor}

Tese apresentada como requisito parcial para obtenção do grau de Doutor pelo Programa de Pós-Graduação em Engenharia Mecânica do Centro Técnico Científico da PUCRio. Aprovada pela Comissão Examinadora abaixo assinada.

Prof. José Alberto dos Reis Parise Orientador Pontifícia Universidade Católica do Rio de Janeiro

Prof. José Viriato Coelho Vargas Universidade Federal do Parana

Prof. Carlos Eduardo Reuther de Siqueira Universidade Católica de Petropolis

Prof. Nisio de Carvalho Lobo Brum Universidade Federal do Rio de Janeiro

Prof. Marco Aurélio Cavalcanti Pacheco Pontifícia Universidade Católica do Rio de Janeiro

Prof. Sergio Leal Braga Pontifícia Universidade Católica do Rio de Janeiro

Prof. Frank Chaviano Pruzaesky Pontifícia Universidade Católica do Rio de Janeiro Jose Eugenio Leal Coordenador(a) Setorial do Centro Técnico Científico - PUC-Rio Rio de Janeiro, 28 de Abril de 2009 
Todos os direitos reservados. É proibida a reprodução total ou parcial do trabalho sem autorização da universidade, do autor e do orientador.

\section{Luis Carlos Castillo Martínez}

Formado como Engenheiro Naval na Universidad Nacional de Ingenieria, Lima, Perú (2002) e como Mestre em Engenharia Mecânica pela PUC-Rio (2004). Envolveu-se na Equipe de Simulação Numérica em Fluidos e Transferência de Calor da UNI (1999), com pesquisa experimental no Laboratório de Refrigeração da PUC-Rio (2003) e em projetos de simulação e otimização de sistemas térmicos utilizando metodologias inteligentes de otimização (PUC-Rio, 2007).

Ficha Catalográfica

Castillo Martínez, Luis Carlos

Otimização dos circuitos de refrigerante nos trocadores de calor de sistemas de refrigeração por compressão de vapor / Luis Carlos Castillo Martínez ; orientador: José Alberto dos Reis Parise ; co-orientadores: Samuel Fortunato Yana Motta, Elizabet del Carmen Vera Becerra. - 2009.

247 f. : il. ; $30 \mathrm{~cm}$

Tese (Doutorado em Engenharia Mecânica)Pontifícia Universidade Católica do Rio de Janeiro, Rio de Janeiro, 2009.

Inclui bibliografia

1. Engenharia mecânica - Teses. 2. Otimização. 3. Simulação. 4. Sistemas de refrigeração por compressão de vapor. 5. Circuito de refrigerante. 6. Algoritmos Genéticos. 7. Trocadores de calor de tipo tubo-aletado. 8. Condensadores de microcanais. I. Parise, José Alberto dos Reis. II. Motta, Samuel Fortunato Yana. III. Vera Becerra, Elizabet del Carmen. IV. Pontifícia Universidade Católica do Rio de Janeiro. Departamento de Engenharia Mecânica. V. Título. 


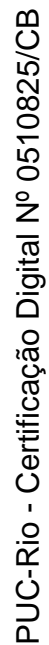

A Nosso Senhor,

A meus pais, Luis e Natalia,

A minha Abi, pelo amor, apoio e confiança. 


\section{Agradecimentos}

$\mathrm{Na}$ verdade muita gente ajudou a completar este trabalho. Em primeiro lugar gostaria de agradecer ao meu orientador, o Prof. José Alberto Parise, pela paciência e pela confiança depositada e, sobretudo, pelos sábios conselhos em diferentes situações que me tocou sortear.

Também gostaria de agradecer à Honeywell International, Inc. USA., de maneira particular a Samuel e Elizabet, por seu apoio e pela oportunidade de nos brindar um caso de estudo prático, de interesse e aplicação direta para a indústria, o qual foi sinônimo de motivação para o desenvolvimento deste trabalho.

Aos meus caros companheiros de estudos do Laboratório de Refrigeração da PUC-Rio, pelas horas compartilhadas, os quais estiveram sempre prontos a me apoiar.

Gostaria de terminar fazendo uma menção especial à bibliotecária Mônica Oliveiros pela simpatia e pela enorme ajuda emprestada ao longo de minha pesquisa.

Agradecimento em particular para o órgão de fomento à pesquisa, $\mathrm{CNPq}$, pelo apoio fornecido, sem o qual este trabalho simplesmente não teria sido possível. 


\section{Resumo}

Martínez, Luis Castillo; Parise, José Alberto Reis. Otimização dos Circuitos de Refrigerante nos Trocadores de Calor de Sistemas de Refrigeração por Compressão de Vapor. Rio de Janeiro, 2009. 247p. Tese de Doutorado - Departamento de Engenharia Mecânica, Pontifícia Universidade Católica do Rio de Janeiro.

Em sistemas de refrigeração por compressão de vapor, o projeto adequado dos circuitos para o refrigerante nos trocadores de calor pode ter um impacto significativo no seu coeficiente de performance (COP). O projeto otimizado dos circuitos de refrigerante em sistemas de refrigeração com trocadores de calor do tipo tubo-aletado não é trivial, devido à complexidade de sua representação assim como o elevado número de possíveis combinações, mesmo quando metodologias inteligentes de otimização são empregadas. No presente trabalho propõe-se uma nova metodologia para a otimização simultânea (condensador e evaporador) dos circuitos do refrigerante em sistemas de refrigeração com trocadores de calor de tipo tubo-aletado. Esta metodologia, aqui denominada como GAFIS (Genetic algorithms applied in filtered spaces), mostra-se mais eficiente que as metodologias até então descritas na literatura. Foi aplicado o método GAFIS, em conjunto com um simulador completo para o sistema de refrigeração, Genesym, na otimização de unidades comerciais de condicionamento de ar de alto desempenho. Estudaram-se casos onde o sistema atingiu aumentos de até $15,3 \%$ no coeficiente de performance. Em outros estudos, obtiveram-se casos onde o custo de produção foi reduzido em $3,85 \%$ (do custo total da unidade), mantendo-se um similar desempenho (capacidade e COP). Testes de otimização, considerando-se diferentes diâmetros dos tubos, na construção dos trocadores de calor, e sistemas com distribuição não uniforme de velocidade de ar, também foram realizados com o GAFIS. Igualmente foram estudados condensadores de microcanais, devido ao interesse atual da indústria com estes trocadores de calor. A otimização do circuito para o refrigerante, neste caso, é relativamente simples, devido ao baixo custo computacional das simulações. Entretanto, modelos de simulação adequados para estes tipos de trocadores de calor só recentemente começaram a surgir, e não têm sido explorados de maneira adequada até a presente data. Explorou-se no presente trabalho, a influência, no desempenho térmico do condensador, dos parâmetros que definem o circuito do refrigerante. Para tal 
efeito, desenvolveu-se um modelo de simulação baseado em análise local, validado com dados experimentais disponíveis, de condensadores de microcanais de uso automotivo com diâmetro hidráulico (lado do refrigerante) de 0,9 e 1,0mm, para refrigerantes R-134a, Fluid-H e R-1234yf. Foram encontradas relações diretas entre os parâmetros geométricos que definem os circuitos de refrigerante no condensador e seu desempenho térmico. Tal fato pode ser utilizado como orientação expedita para o projeto do circuito ótimo do condensador.

\section{Palavras-chave}

Otimização, Simulação, Sistemas de refrigeração por compressão de vapor, Circuito de refrigerante, Algoritmos Genéticos, Trocadores de calor de tipo tubo-aletado, Condensadores de microcanais. 


\section{Abstract}

Martínez, Luis Castillo; Parise, José Alberto Reis (Advisor). Optimization the circuiting refrigeration of the heat exchangers in Vapor Compression Refrigeration Systems. Rio de Janeiro, 2009. 247p. D.Sc. Thesis - Departamento de Engenharia Mecânica, Pontifícia Universidade Católica do Rio de Janeiro.

Refrigerant circuiting in condensers and evaporators has a significant effect in the performance of refrigeration systems. The optimized project of the refrigerant circuits in refrigeration systems with plate-fin heat exchangers is not trivial, due to the complexity of their representation as well as the high number of possible combinations, even when methodologies of intelligent optimization are used. The present work proposes a new methodology for the simultaneous optimization of refrigerant circuiting in air-air refrigeration systems with plate-fin heat exchangers. This new methodology, here defined as GAFIS (Genetic algorithms applied in filtered spaces), has proven to be more efficient than traditional methods. The GAFIS method was applied, in conjunction with a full refrigeration system simulator, Genesym, for the optimization of high performance commercial air-conditioning units. Typical cases were studied and a coefficient of performance improvement of up to $15.3 \%$ has been observed. In other studies, there were cases where the manufacturer's predicted cost was reduced in $3,85 \%$ (of total cost of the unit), while a similar thermal performance (capacity and COP) was maintained. Optimization tests, considering different diameters of tube, for the construction of heat exchangers, as well as systems with non-uniform air velocity distribution, were also performed with the GAFIS method. Microchannel condensers were also studied, given the current interest of industry on this kind of heat exchanger. The optimization of the refrigerant circuiting, in this case, would not be a major problem, due to the low computational cost of its simulation. However, simulation models appropriate for these types of heat exchangers have only been recently in use, and, to date, have not been adequately explored. In the present work, the influence on condenser performance of parameters that define the refrigerant circuiting has been investigated. For this purpose, a simulation model, based on local analysis, was developed. It was validated against experimental data, available from automotive microchannel condenser tests, with hydraulic diameters (refrigerantside) of 0.9 and $1.0 \mathrm{~mm}$ for refrigerants R-134a, Fluid-H and R-1234yf. A direct 
relation was found between the geometric parameters that define the condenser refrigerant circuiting and its thermal performance. This fact can be appropriately used as guidance for expeditious design practices of the optimal refrigerant circuit of the condenser.

\section{Keywords}

Optimization, Simulation, Vapor-Compression Refrigeration System, Refrigerant circuits, Genetic Algorithms, Finned Tube Heat Exchanger, Microchannels condenser. 


\section{Sumário}

1. Introdução 26

1.1. Motivação 28

1.1.1. Redução do impacto ambiental 28

1.1.2. Otimização energética em sistemas de refrigeração 29

1.1.3. Falta de um método eficiente de otimização para o circuito do refrigerante em trocadores de calor de tipo tubo-aletado $\quad 29$

1.1.4. Tendência ao uso de trocadores de calor de microcanais 29

1.1.5. Falta de um método de busca do circuito ótimo em um trocador de calor de microcanais $\quad 30$

1.1.6. Potencial de uso com a chegada de novos refrigerantes 30

1.2. Revisão Bibliográfica $\quad 30$

1.2.1. Simulação de sistemas de refrigeração 30

1.2.2. Simulação de trocadores de calor de tipo tubo-aletado 31

1.2.3. Otimização de trocadores de calor tipo tubo-aletado 31

1.2.4. Trocadores de calor de microcanais 36

1.3. Objetivos e Contribuições $\quad 36$

1.3.1. Nova metodologia de otimização 36

1.3.2. Otimização simultânea dos dois trocadores de calor de tipo tubo-aletado 37

1.3.3. Simulação e otimização de Condesadores de Microcanais 37

1.4. Estrutura do Trabalho $\quad 37$

2. Métodos de otimização 40

2.1. Introdução 40

2.1.1. Conceitos gerais $\quad 41$

2.1.2. Representação matemática de um problema de otimização 42

2.2. Classificação dos Métodos de Otimização 42

2.2.1. Métodos Determinísticos $\quad 42$

2.2.2. Métodos Probabilísticos 43

2.3. Computação Evolucionária 45

2.3.1. Programação evolutiva 46

2.3.2. Estratégias evolutivas 46

2.3.3. Programação genética $\quad 47$

2.3.4. Algoritmos genéticos $\quad 47$ 
2.4. Comentários sobre os métodos de otimização e o problema de otimização de sistemas de refrigeração com TCTA's através dos circuitos 47

2.5. Método dos Algoritmos Genéticos 49

2.5.1. Introdução 49

2.5.2. Conceitos gerais $\quad 50$

2.5.3. Princípio básico de um Algoritmo Genético 52

2.5.4. Operadores Genéticos 53

2.5.4.1. Operador de Cruzamento $\quad 54$

2.5.4.2. Operador de Mutação 54

2.5.5. Parâmetros do Método dos Algoritmos Genéticos 55

2.5.5.1. Tamanho da população 55

2.5.5.2. Probabilidade de Cruzamento 55

2.5.5.3. Probabilidade de Mutação 56

3. Simulação de Sistemas de Refrigeração por Compressão de Vapor 57

3.1. Introdução $\quad 57$

3.2. Revisão bibliográfica $\quad 59$

3.3. Simulador Genesym $\quad 65$

3.3.1. Dados de entrada $\quad 67$

$\begin{array}{ll}\text { 3.3.2. Método de solução } & 73\end{array}$

$\begin{array}{ll}\text { 3.3.3. Dados de saída } & 74\end{array}$

4. Metodologia de otimização 76

4.1. Introdução 76

4.2. Descrição do problema $\quad 77$

$\begin{array}{ll}\text { 4.2.1. Objetivo da otimização } & 77\end{array}$

4.2.2. Variáveis do problema $\quad 78$

4.2.3. Restrições 78

4.3. Definição do número ótimo de ramais nos trocadores de calor 79

4.4. Nova metodologia de otimização (GAFIS) 79

4.4.1. Algoritmo (GAFIS)

4.4.2. Representação dos circuitos

4.4.3. Filtro de restrições e Geração do banco de dados 85

4.4.4. Operadores Genéticos 86

4.5. Ajuste dos parâmetros para o método de AG 87

4.6. Validação do método 9

4.6.1. Comparação com o método de busca aleatória 90

4.6.2. Qualidade das soluções 92 
5.1. Caso 1: Melhora do desempenho de um Sistema tipo Mini-Split de 3 TR da ICP

5.1.1. Dados de entrada $\quad 95$

5.1.2. Testes realizados $\quad 98$

5.1.3. Evolução dos parâmetros no ciclo de refrigeração 103

5.1.4. Análise Exergética do Ciclo Otimizado 106

5.2. Caso 2: Diminuição do custo de produção de um Sistema tipo Split de

3,2 TR da Lennox 109

5.2.1. Dados de entrada 109

5.2.2. Testes realizados 112

5.2.3. Análise dos custos de produção 115

$\begin{array}{ll}\text { 5.2.4. Análise Exergética do Ciclo Otimizado } & 117\end{array}$

5.3. Caso 3: Estudo de sensibilidade com respeito ao uso de diâmetros menores nos equipamentos de condicionamento de ar 119

5.3.1. Dados de entrada 119

$\begin{array}{ll}\text { 5.3.2. Testes realizados } & 123\end{array}$

5.3.3. Evolução dos parâmetros do ciclo de refrigeração 128

5.3.4. Análise Exergética dos Ciclos Otimizados $\quad 130$

5.4. Caso 4: Melhora do desempenho de um Sistema tipo Split de 0,9 TR da Haier com distribuição de ar não uniforme 134

5.4.1. Dados de entrada 134

$\begin{array}{ll}\text { 5.4.2. Testes realizados } & 138\end{array}$

5.4.3. Evolução dos parâmetros do ciclo de refrigeração 142

5.4.4. Análise Exergética do Ciclo Otimizado 144

6. Simulação e Otimização de Condensadores de Microcanais 146

6.1. Revisão Bibliográfica 146

6.2. Modelagem de um Condensador de Microcanais 151

6.2.1. Descrição de um Condensador de Microcanais 151

6.2.2. Descrição do Modelo de Simulação 152

6.2.3. Análise de um Elemento de Controle 154

6.2.3.1. Balanço de Energia 154

6.2.3.2. Áreas de transferência de calor e efetividade das aletas 155

6.2.3.3. Queda de pressão 156

6.2.4. Modelos teóricos implementados 157

6.2.4.1. Modelos de transferência de calor no lado do refrigerante 157

6.2.4.2. Modelos de transferência de calor no lado do ar 160

$\begin{array}{ll}\text { 6.2.4.3. Modelos de queda de pressão no lado do refrigerante } & 161\end{array}$ 
6.2.4.4. Modelos para a queda de pressão no lado do ar 164

6.2.5. Metodologia de Solução 165

6.2.6. Dados de entrada e saída do programa 169

6.2.6.1. Dados de entrada 169

6.2.6.2. Dados de saída 170

6.2.7. Teste de discretização 172

6.3. Validação com dados experimentais 173

6.3.1. Plano de Testes realizado 173

6.3.2. Dados experimentais 173

6.3.2.1. Dados experimentais para o CD1 utilizando R134a e Fluid-H 174

6.3.2.2. Dados experimentais para o CD2 utilizando R134a e 1234yf 175

6.3.2.3. Comentários sobre os dados experimentais 177

6.3.3. Comentários das comparações com os dados experimentais 178

6.4. Circuito ótimo num condensador de microcanais 186

6.4.1. Abordagem utilizada 186

6.4.1.1. Representação e restrições de um circuito 186

$\begin{array}{lc}\text { 6.4.1.2. Metodologia utilizada } & 188\end{array}$

6.4.2. Resultados da busca exaustiva 189

6.4.3. Análise de resultados 192

6.4.4. Circuito ótimo para o CD1 na condição 07LSH45 195

$\begin{array}{ll}\text { 7. Conclusões } & 199\end{array}$

8. Recomendações e Sugestões 205

$\begin{array}{ll}\text { Bibliografia } & 208\end{array}$

Apêndice A - Análise Exergética 229

Apêndice B - Correlações do Genesym 233

Apêndice C - Resumo de características para os testes realizados 239

$\begin{array}{ll}\text { Apêndice D - Estudos referentes a microcanais } & 240\end{array}$ 


\section{Lista de tabelas}

Tabela 1. Resumo dos dados de entrada necessários para o processo de simulação.

Tabela 2. Resumo dos dados de saída providos pelo Simulador Genesym. $\quad 74$

Tabela 3. Resumo dos testes utilizando diferentes parâmetros de otimização. $\quad 90$

Tabela 4. Resumo de valores adequados para os parâmetros do GAFIS. 90

Tabela 5. Resumo de testes utilizando os mesmos parâmetros de otimização. $\quad 93$

Tabela 6. Dados gerais do projeto AC-ICP-3TON-A (Sistema da ICP). 97

Tabela 7. Configurações dos circuitos nos testes C1T1, C1T2 e C1T3. 99

Tabela 8. Resumo de resultados dos testes de otimização na Unidade da ICP. 102

Tabela 9. Análise de irreversibilidades do sistema original e do sistema

otimizado no teste C1T3. 106

Tabela 10. Dados gerais do projeto b010330k (Sistema da Lennox). 111

Tabela 11. Configurações dos circuitos nos testes C2T1 e C2T2. 112

Tabela 12. Resumo de resultados dos testes de otimização no sistema Lennox. 114

Tabela 13. Custo de material e de fabricação considerados. 115

Tabela 14. Custos de fabricação da unidade original e das unidades otimizadas. 116

Tabela 15. Análise de irreversibilidades, sistema original - sistema otimizado. 117

Tabela 16. Dados gerais do projeto a001208a-case01mod (Sistema da Lennox). 121

Tabela 17. Dados gerais do projeto a001208a-case02mod (Sistema da Lennox). 121

Tabela 18. Dados gerais do projeto a001208a-case03mod (Sistema da Lennox). 122

Tabela 19. Dados gerais do projeto a001208a-case04mod (Sistema Lennox). 122

Tabela 20. Configuração utilizada para os circuitos no teste C3T1 ao C3T4. 124

Tabela 21. Configuração utilizada para os circuitos no teste C3T5 ao C3T8. 124

Tabela 22. Resumo de resultados dos testes de otimização do sistema Lennox

$\begin{array}{ll}\text { para R22. } & 127\end{array}$

Tabela 23. Resumo de resultados dos testes de otimização do sistema Lennox $\begin{array}{ll}\text { para R410A. } & 127\end{array}$

Tabela 24. Análise de irreversibilidades, sistema original - sistema otimizado. 130

Tabela 25. Análise de irreversibilidades, sistema original - sistema otimizado. 132

Tabela 26. Dados gerais do projeto R22-9580base (Sistema da Haier). 137

Tabela 27. Configurações dos circuitos nos testes C4T1 e C4T2. 138

Tabela 28. Resumo de resultados dos testes de otimização do sistema Haier. 141

Tabela 29. Análise de irreversibilidades, sistema original - sistema otimizado. 144

Tabela 30. Expressões relativas à transferência de calor. 154

Tabela 31. Correlações para o cómputo da efetividade da superfície aletada. 156 
Tabela 32. Componentes para a queda de pressão.

Tabela 33. Número de Nusselt para fluxo monofásico no lado do refrigerante.

Tabela 34. Correlações para o número de Nusselt na zona bifásica no lado do refrigerante.

Tabela 35. Modelo para o fator de Coulburn no lado do ar.

Tabela 36. Correlações para o fator de atrito em escoamento monofásico.

Tabela 37. Modelos para a queda de pressão bifásica no lado do refrigerante. 163

Tabela 38. Modelo para o cálculo do coeficiente de atrito no lado do ar.

Tabela 39. Condições de entrada para um elemento genérico de controle.

Tabela 40. Dados de entrada considerados necessários para a simulação do condensador de microcanais.

Tabela 41. Combinações de modelos teóricos para a transferência de calor e queda de pressão que foram utilizadas nas simulações.

Tabela 42. Dados experimentais para o CD1, utilizando R134a.

Tabela 43. Dados experimentais para o CD2, utilizando R134a.

Tabela 44. Detalhe da instrumentação utilizada e sua precisão.

Tabela 45. Estatísticas de erro de comparação dos modelos teóricos, para o CD1 utilizando R134a.

Tabela 46. Estatísticas de erro de comparação dos modelos teóricos, para o CD1 utilizando Fluid-H.

Tabela 47. Estatísticas de erro de comparação dos modelos teóricos, para o CD2 utilizando R134a.

Tabela 48. Estatísticas de erro de comparação dos modelos teóricos, para o CD2 utilizando 1234yf.

Tabela 49. Resultados da simulação numérica, para diversos arranjos de circuitos no CD1 com R134a, para a condição 07LSH45. 


\section{Lista de figuras}

Figura 1. Principais algoritmos da Computação Evolucionária (Adaptado de Holtz, 2005).

Figura 2. Ramos da Inteligência Artificial (Holtz, 2005).

Figura 3. Diagrama de Fluxo básico de um Algoritmo Genético. 52

Figura 4. Princípio básico de um algoritmo genético (Pacheco, 2006). 53

Figura 5. Exemplo de aplicação de um operador de cruzamento. 54

Figura 6. Exemplo de aplicação de um operador de mutação. 55

Figura 7. Ciclo de refrigeração por compressão de vapor no diagrama P-h. $\quad 58$

Figura 8. Interface gráfica do Simulador Genesym v1.0. 68

Figura 9. Dados geométricos necessários para definir um trocador de calor. $\quad 68$

Figura 10. Geometria do Trocador de Calor no Genesym. 69

Figura 11. Seleção das junções entre os tubos para definir o circuito e definição do perfil de velocidade para o ar (no caso, uniforme). $\quad 70$

Figura 12. Dados necessários para definir o compressor.

Figura 13. Detalhes para definir a conexão da linha de sucção. 71

Figura 14. Condições de operação e modo de avaliação do ciclo. 72

Figura 15. Exemplo de resultados tubo-a-tubo com queda de pressão no lado do refrigerante. $\quad 75$

Figura 16. Diagrama de fluxo do processo de otimização - GAFIS. 82

Figura 17. Sistema de condicionamento de ar com trocadores de calor de tipo tubo-aletado. 83

Figura 18. Representação dos circuitos. $\quad 84$

Figura 19. Filtragem de soluções para gerar os bancos de dados. 85

Figura 20. Efeito dos operadores genéticos. $\quad 87$

Figura 21.a Evolução do COP médio para a população de soluções. 89

Figura 21.b Evolução da dispersão do COP para a população de soluções. $\quad 89$

Figura 22. Maximo COP obtido com GAFIS versus o método de busca aleatória. 91

Figura 23. Convergência de soluções pelo GAFIS. 92

Figura 24. Evaporador do Sistema Mini-Split de 3 TR da ICP. 96

Figura 25. Unidade Condensadora do Sistema Mini-Split de 3 TR da ICP. 96

Figura 26. Circuitos originais do refrigerante no sistema da ICP. 96

Figura 27. Teste C1T1 - Circuitos otimizados considerando a configuração base. 99

Figura 28. Teste C1T2 - Circuitos otimizados para a Unidade da ICP. 100

Figura 29. Teste C1T3 - Circuitos otimizados para a Unidade da ICP. 101

Figura 30. Teste C1T4 - Circuitos otimizados para a Unidade da ICP. 101 
Figura 31. Test C1T2 - Evolução dos principais parâmetros de funcionamento do ciclo de refrigeração durante a otimização.

Figura 32. (a) Irreversibilidades no sistema, (b) Variação no ciclo derefrigeração. 108 Figura 33. Unidade evaporadora para o sistema comercial da Lennox - modelo HSX19.

Figura 34. Unidade condensadora para o sistema comercial da Lennox - modelo HSX19.

Figura 35. Circuitos originais do sistema Lennox.

Figura 36. Teste C2T1 - Circuitos otimizados considerando a configuração base 113

Figura 37. Teste C2T2 - Circuitos otimizados do caso Lennox. 114

Figura 38. (a) Irreversibilidades no sistema, (b) Variação no ciclo de refrigeração 118

Figura 39. Circuitos originais do sistema Lennox (projeto a001208a-mod). 119

Figura 40. Teste C3T1 - Circuitos otimizados (a001208a-mod com R22). 125

Figura 41. Teste C3T2 - Circuitos otimizados (a001208a-mod com R22). 125

Figura 42. Teste C3T3 - Circuitos otimizados (a001208a-mod com R22). 125

Figura 43. Teste C3T4 - Circuitos otimizados (a001208a-mod com R22). 125

Figura 44. Teste C3T5 - Circuitos otimizados (a001208a-mod com R410A). 126

Figura 45. Teste C3T6 - Circuitos otimizados (a001208a-mod com R410A). 126

Figura 46. Teste C3T7 - Circuitos otimizados (a001208a-mod com R410A). 126

Figura 47. Teste C3T8 - Circuitos otimizados (a001208a-mod com R410A). 126

Figura 48. Teste C3T1 - Evolução dos principais parâmetros de funcionamento do ciclo de refrigeração durante a otimização.

Figura 49. (a) Irreversibilidades no sistema, (b) Variação no ciclo de refrigeração 131 Figura 50. (a) Irreversibilidades no sistema, (b) Variação no ciclo de refrigeração 133 Figura 51. Esquema da Bomba de Calor da Haier. 135

Figura 52. Esquema típico de um sistema de condicionamento de ar tipo "split". 135 Figura 53. Fotografias do (a) Evaporador e (b) Condensador do sistema Haier de 0,9 TR.

Figura 54. Circuitos originais do sistema Haier (projeto R22-9580base).

Figura 55. Perfil de velocidade adimensional no trocador de calor interno (evaporador).

Figura 56. Perfil de velocidade adimensional no trocador de calor externo (condensador).

Figura 57. Teste C4T1 - Circuitos otimizados deixando tubos sem utilizar. $\quad 139$

Figura 58. Teste C4T2 - Circuitos otimizados utilizando todos os tubos. $\quad 140$

Figura 59. Teste C4T3 - Teste com condensador desbalanceado. 140

Figura 60. Teste C4T3 - Teste com ramal de subresfriamento no condensador. 140

Figura 61. Teste C4T3 - Teste deixando tubos sem utilizar no condensador. 141 
Figura 62. C4T2 - Evolução dos principais parâmetros de funcionamento do ciclo de refrigeração durante a otimização.

Figura 63. (a) Irreversibilidades no sistema, (b) Variação no ciclo de refrigeração 145

Figura 64. Condensador de microcanais de uso automotivo.

Figura 65. Detalhe das aletas com persianas num condensador de microcanais 152

Figura 66. Elemento básico de análise.

Figura 67. Dimensões geométricas envolvidas na definição das áreas de transferência de calor num elemento básico de controle.

Figura 68. Geometria das aletas com persianas (adaptado de Chang et al. 2006) 165

Figura 69. Sistema de coordenadas considerado para a Matriz de elementos de controle.

Figura 70. Definição do circuito e acompanhamento do fluxo do refrigerante. 166

Figura 71. Algoritmo para a solução do condensador. 169

Figura 72. Variação de parâmetros ao longo do condensador (07LSH35). 171

Figura 73. Variação do título e campo de temperatura para o ar (Test.07LSH35). 171

Figura 74. Análise de sensibilidade na solução do sistema (Test.07 LSH35). 172

Figura 75. Geometria do Condensador CD1. 174

Figura 76. Geometria do Condensador CD2. 175

Figura 77. Esquemas da câmara controlada de climatização e da bancada experimental.

Figura 78. Comparação entre valores previstos pelas simulações e os dados experimentais, para o CD1 utilizando R134a.

Figura 79. Comparação entre valores previstos pelas simulações e os dados experimentais, para o CD1 utilizando Fluid-H.

Figura 80. Comparação entre valores previstos pelas simulações e os dados experimentais, para o CD2 utilizando R134a.

Figura 81. Comparação entre valores previstos pelas simulações e os dadosexperimentais, para o CD2 utilizando 1234yf.

Figura 82. Múltiplas possibilidades de arranjo para o circuito, considerando n passes.

Figura 83. Algoritmo para a exploração das possibilidades de desenho do circuito.

Figura 84. Variação da Taxa de Transferência de Calor e da Queda de Pressão em função do número de passes.

Figura 85. Variação da Taxa de Transferência de Calor e da Queda de Pressão dentro de um grupo com igual número de passes. 
Figura 86. Variação da Taxa de Transferência de Calor e da Queda de Pressão dentro de um subgrupo com igual número de passes e de flats nos quatro primeiros passes.

Figura 87. Taxa de transferência de calor e queda de pressão encontradas nas simulações em torno do valor capacidade-queda de pressão do circuito original. 196 Figura 88. Ampliação da fig.87 - com respeito à taxa de transferência de calor e à queda de pressão nas simulações em torno do valor capacidade-queda de pressão original. 


\section{Lista de símbolos}

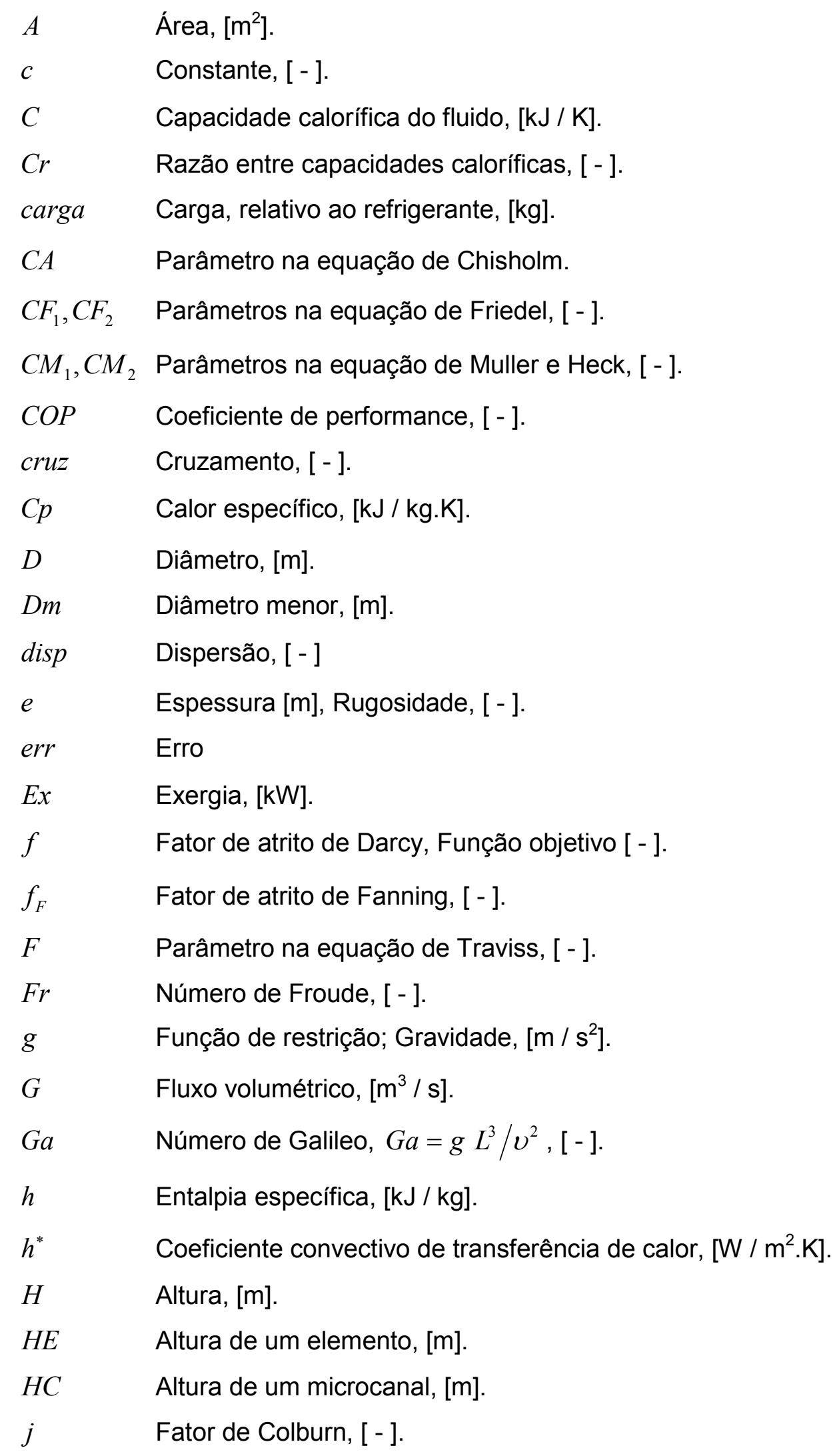


$k \quad$ Condutividade térmica, [kW / m.K].

$K_{c}, K_{e} \quad$ Coeficientes de expansão e contração abrupta, [ - ].

L Comprimento, [m].

LE Comprimento de um elemento, [m].

m Vazão mássica, $[\mathrm{kg} / \mathrm{s}]$.

$M \quad$ Massa molar, [kg / mol].

$M A P E \quad$ Erro médio percentual absoluto, [\%].

MPE Erro médio percentual, [\%].

mut Mutação, [ - ].

$m_{\text {alt }} \quad$ Parâmetro para o cálculo da efetividade da aleta, [ - ].

$n \quad$ Expoente de Blasius, [ - ]; Indicador de numeral, [ - ].

Nc Número de microcanais por elemento, [ - ].

NTU Número de unidades de transferência de calor, [ - ].

Número de Nusselt, $N u=h^{*} D / k,[-]$.

$\operatorname{Pr}$

Número de Prandtl, $\operatorname{Pr}=\mu C p / k,[-]$.

$P \quad$ Perímetro, [m].

p Pressão, [kPa].

Ps Passo, $[\mathrm{m}]$.

$\dot{Q} \quad$ Taxa de transferência de calor, [kW].

$R \quad$ Fator de correlação, [\%].

$\operatorname{Re} \quad$ Número de Reynolds, $\operatorname{Re}=\rho \cdot v \cdot D / \mu,[-]$.

$R M S P E \quad$ Erro quadrático médio percentual, [\%].

$S$

Entropia específica, [kJ / kg.K].

$T \quad$ Temperatura, $[\mathrm{K}]$.

$t \quad$ Espessura, [m]; Tempo, [s].

$U \quad$ Coeficiente global de transferência de calor, [kW/ $\left.\mathrm{m}^{2} . \mathrm{K}\right]$.

$U A \quad$ Condutância total, [kW / K].

$V \quad$ Volume, $\left[\mathrm{m}^{3}\right]$.

w Fator de ponderação na equação de Chang, [ - ].

W Trabalho, [kW]; Largura [m].

We Número de Webber, [ - ].

WE Largura externa do Elemento, [m].

WI Largura interna do Elemento, [m]. 

WB Largura do Webb, [m].
$x \quad$ Variável de projeto, Titulo, [ - ].
$\chi_{t t} \quad$ Parâmetro de Martinelli, [ - ].
Y Parâmetro na equação de Chisholm, [ - ].

\section{Símbolos gregos}

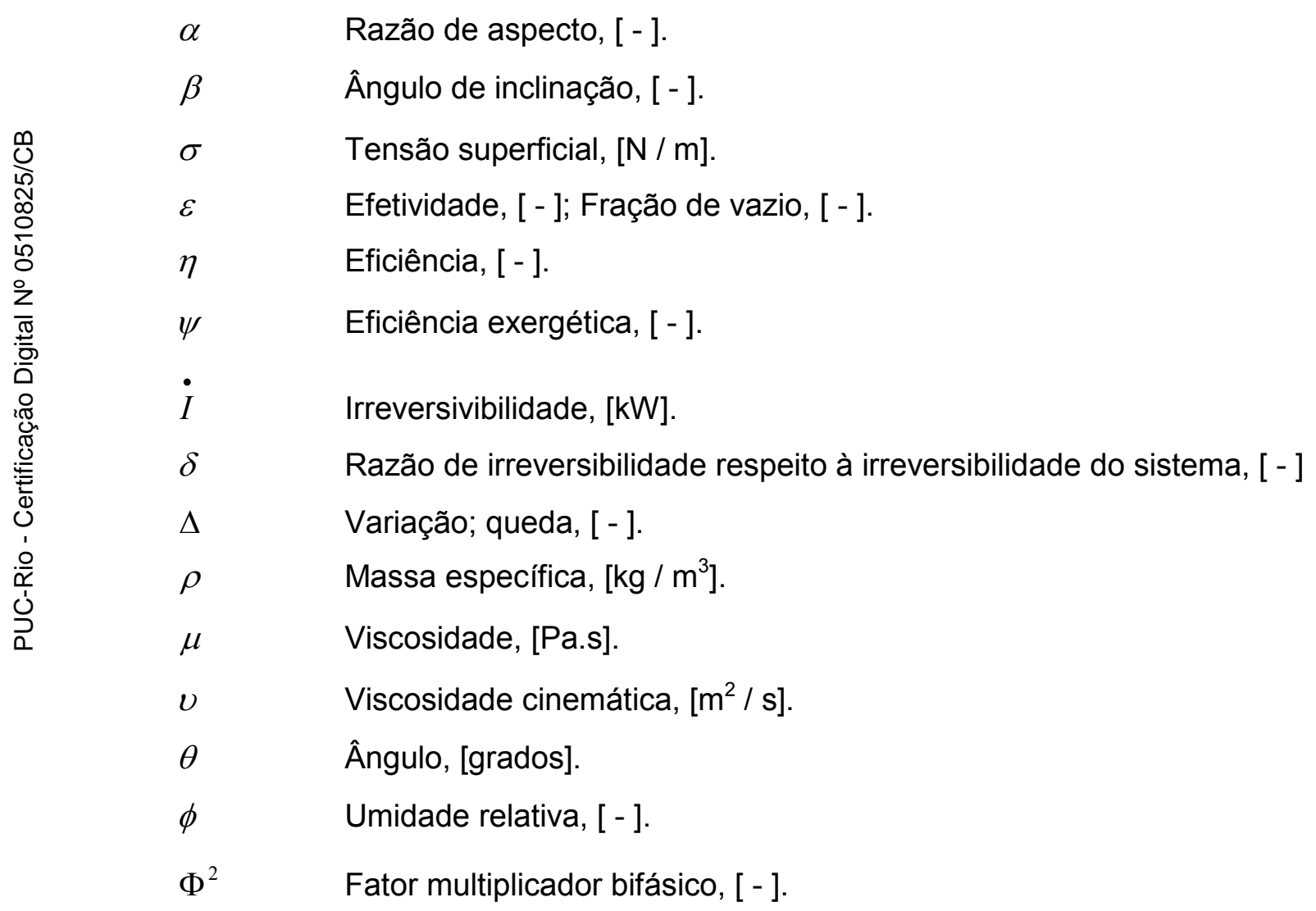




\section{Subscritos}

\begin{tabular}{|c|c|c|}
\hline & air & $\mathrm{Ar}$ \\
\hline & alt & Aleta \\
\hline & acum & Acumulativo \\
\hline & anul & Anular \\
\hline & $c$ & Canal \\
\hline & $c d$ & Condensador \\
\hline & cond & Condensação \\
\hline & comp & Compressor \\
\hline & des & Descarga \\
\hline & $e q$ & Equivalente \\
\hline & $e v$ & Evaporador \\
\hline & evap & Evaporação \\
\hline $\begin{array}{l}\text { ond } \\
\text { o }\end{array}$ & $\exp$ & Experimental \\
\hline $\begin{array}{l}\text { Oे } \\
\text { ol }\end{array}$ & fric & Atrito \\
\hline 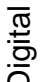 & $f z$ & Forçado \\
\hline 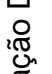 & Go & Somente gás \\
\hline & $h$ & Homogêneo, Hidráulico \\
\hline & in & Entrada \\
\hline & $l$ & Líquido \\
\hline & Lo & Somente líquido \\
\hline & $m$ & Médio (a) \\
\hline & $\min$ & Mínimo \\
\hline & $\max$ & Máximo, [ - ]. \\
\hline & med & Média, [ - ]. \\
\hline & mom & Momentum \\
\hline & orig & Original \\
\hline & out & Saída \\
\hline & per & Persiana \\
\hline & pred & Previsto \\
\hline & $r$ & Reduzida \\
\hline & ref & Refrigerante \\
\hline
\end{tabular}




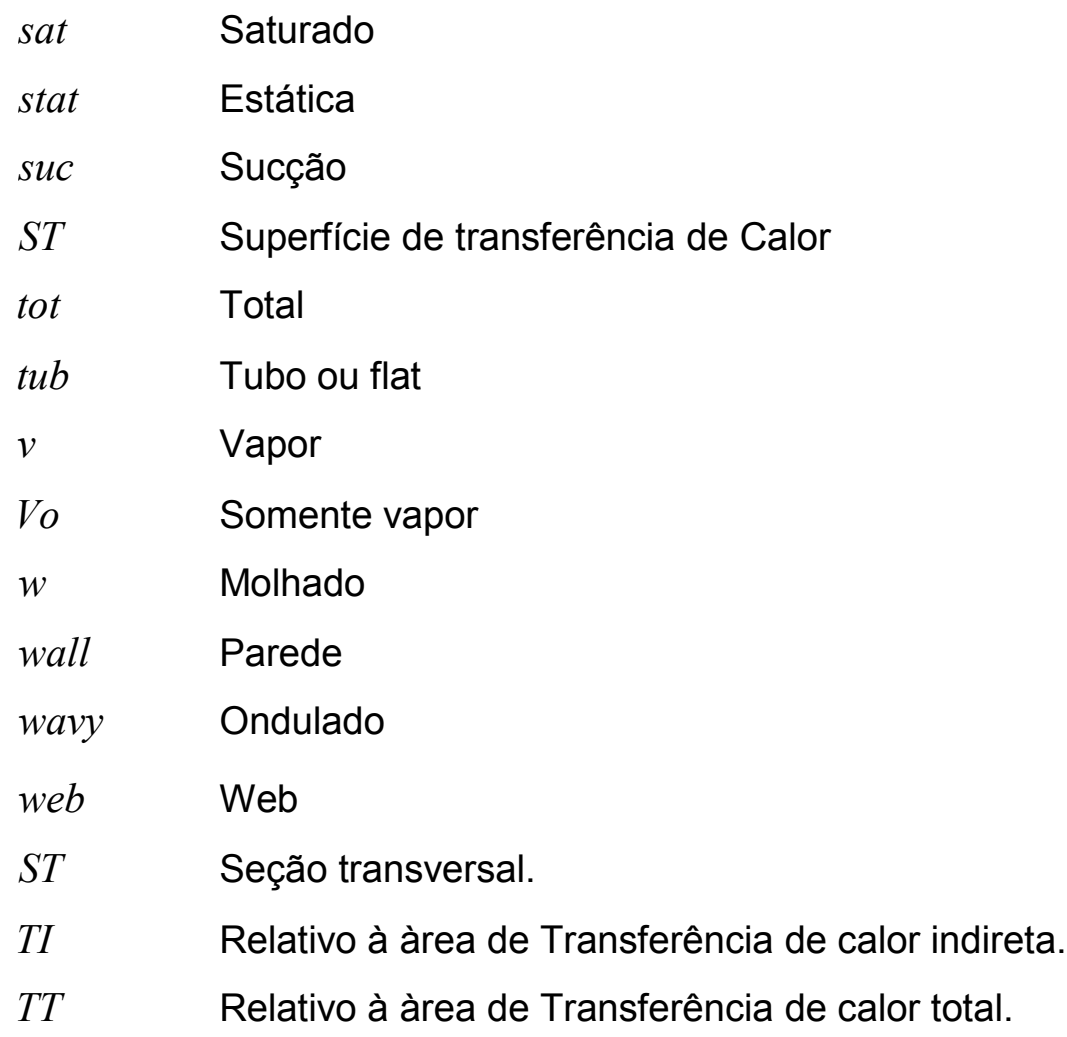


Es común afirmar que Newton habría dicho que si él alcanzaba a ver más lejos que sus contemporáneos no era porque tuviese méritos especiales, no hacía más que apoyarse en el trabajo de otros, como un enano que se alza en hombros de un gigante.

Exactamente eso es lo que nos brinda los estudios, un punto diferente de partida para abrir nuevos horizontes. 